\title{
La escritura académica en contexto: posibilidad de desarrollo profesional de profesores universitarios ${ }^{\star}$
}

\author{
ELIZABETH HERNÁNDEZ VARGAS** \\ MARTHA LILIANA MARÍN CANO***
}

Recepción: 21 de julio de 2017

Aprobación: 28 de mayo de 2018

Forma de citar este artículo: Hernández, E. \& Marín, M. L. (2018). La escritura académica en contexto: posibilidad de desarrollo profesional de profesores universitarios. Cuadernos de Lingüística Hispánica, (32), 61-84

\footnotetext{
* Artículo de revisión.

** Candidata a doctora del programa de Doctorado de Educación de la Universidad Católica de Manizales. Magíster en Lingüística Española del Instituto caro y Cuervo. Investigadora del grupo GIPIS Universidad Cooperativa de Colombia, sede Villavicencio. Correo electrónico: elizaherv@gmail.com

*** Doctora en Ciencias de la Educación de la Red de Universidades Estatales de Colombia. Magíster en Educación del convenio Universidad de Caldas-Universidad Javeriana. Investigadora del grupo ALFA de la Universidad Católica de Manizales. Correo electrónico: mlmarin@ucm.du.co
} 


\section{Resumen}

La investigación a la que se hace referencia en el presente artículo, corresponde a los resultados de un análisis documental (Galeano, 2012) de "La escritura académica en contexto: posibilidad de desarrollo profesional de los profesores universitarios". El estudio pretende, por un lado, identificar a través de las investigaciones en escritura académica las diversas perspectivas teóricas y metodológicas, y, por otro lado, analizar la relación que existe entre escritura académica y el desarrollo profesional del profesor de educación superior. Con esta finalidad, se analizaron 50 documentos que datan del año 2006 hasta el año 2016 y que han sido publicados en diferentes países, tales como Chile, Colombia, México, Portugal, Argentina, Venezuela, Estados Unidos, España y Canadá. Los resultados muestran el interés de los investigadores por las dificultades que existen en el proceso escritor, la formación escritural del profesor universitario, la escritura como una tarea compleja, el uso de la escritura académica, las explicaciones a los problemas de lectura y escritura en el estudiante dejando de lado a los profesores, y el aprendizaje de los estudiantes y la didáctica de los profesores.

Palabras clave: escritura académica, profesor universitario, desarrollo profesional, educación superior.

\section{Academic Writing in Context: Possibility of Professional Development of University Professors}

\section{Abstract}

The research reported in this article corresponds to the results of a documentary analysis (Galeano, 2012) of "The academic writing in context: possibility of professional development of university professors". The study aims, on the one hand, to identify, through research in academic writing, the different theoretical and methodological perspectives, and, on the other hand, to analyze the relationship that exists between academic writing and the professional development of university professor. To this end, 50 documents dating from 2006 to 2016 were analyzed and published in different countries, such as Chile, Colombia, Mexico, Portugal, Argentina, Venezuela, the United States, Spain and Canada. The results show the interest of the researchers due to the difficulties that exist in the writing process, the scriptural formation of the university professor, the writing as a complex task, the use of academic writing, the explanations to the problems of reading and writing in the student leaving aside teachers, and student learning and teachers' didactics.

Keywords: academic writing, university professor, professional development, university education.

\section{L'écriture académique en contexte : possibilité de développement professionnel des enseignants universitaires}




\section{Résumé}

La recherche à laquelle fait référence cet article, correspond aux résultats d'une analyse documentaire (Galeano, 2012) de "L'écriture académique en contexte: possibilité de développement professionnel des enseignants universitaires ». L'étude vise, d'un côté, identifier les diverses perspectives théoriques et méthodologiques, à travers des recherches en écriture académique. De l'autre côté, elle vise à analyser le rapport existant entre écriture académique et le développement professionnel de l'enseignant universitaire. Ayant ce but, on a analysé 50 documents qui datent de 2006 à 2016, et qui ont été publiés dans de différents pays, tels que Chili, Colombie, Mexique, Portugal, Argentine, Venezuela, États-Unis, Espagne et Canada. Les résultats montrent l'intérêt des chercheurs pour les difficultés qui existent dans les processus d'écrire de l'enseignant universitaire, l'écriture en tant que tâche complexe, l'utilisation de l'écriture académique, les explications aux problèmes des lecture et d'écriture chez l'étudiant laissant de côté les enseignants, l'apprentissage des étudiants et la didactique des enseignants.

Mots clés: écriture académique, enseignant universitaire, développement professionnel, éducation supérieure.

\section{Escrita Acadêmica No Contexto: Possibilidade De Desenvolvimento Profissional De Professores Universitários}

\section{Resumo}

A pesquisa referida neste artigo corresponde aos resultados de uma análise documental (Galeano, 2012) de "Escrita acadêmica em contexto: possibilidade de desenvolvimento profissional de professores universitários". O estudo visa, por um lado, identificar, por meio da pesquisa em escrita acadêmica, as diferentes perspectivas teóricas e metodológicas e, por outro, analisar a relação existente entre a escrita acadêmica e o desenvolvimento profissional do professor de ensino superior. Para este fim, 50 documentos datados de 2006 a 2016 foram analisados e publicados em diferentes países, como Chile, Colômbia, México, Portugal, Argentina, Venezuela, Estados Unidos, Espanha e Canadá. Os resultados mostram o interesse dos pesquisadores pelas dificuldades que existem no processo de escrita, a formação das escrituras do professor universitário, a escrita como uma tarefa complexa, o uso da escrita acadêmica, as explicações para os problemas de leitura e escrita no aluno deixando de lado os professores, e aprendizado dos alunos e didática dos professores.

Palavras-chave: redação acadêmica, professor universitário, desenvolvimento profissional, ensino superior. 


\section{Introducción}

El propósito de esta revisión y análisis documental es identificar los estudios realizados en América, Canadá y Europa sobre la escritura académica en contexto: posibilidad para el desarrollo profesional de los profesores universitarios, primera fase del estudio de carácter doctoral que centra su atención en el desarrollo de la pregunta problema: ¿cómo la potenciación de la escritura académica en contexto fortalece el desarrollo profesional de los profesores universitarios del Programa de Ingeniería Civil de la Universidad Cooperativa de Colombia, sede Villavicencio?

Los documentos estudiados se identificaron a través de la búsqueda de revistas especializadas, bases de datos, memorias de congresos, libros y estados del tema, durante los años 2006 a 2016, para describir, caracterizar, analizar e interpretar la escritura académica en contexto: posibilidad para el desarrollo profesional de los profesores universitarios.

Esta investigación se enmarca en la revisión documental y sus resultados específicos son de tipo cualitativo, ya que parte de "las descripciones, explicaciones y comprensiones que sobre la realidad ha construido una investigación previa. Es decir, que este tipo de estudios oscila entre los referentes más inmediatos a los hechos, los resultados de la investigación previa y la construcción teórica" (Manjarrés \& Vargas, 1998, p. 24). Para su elaboración, se hizo la revisión bibliográfica y el análisis de contenidos digitales, lo que implicó un trabajo de búsqueda rastreando diferentes fuentes de información durante varios meses y la recuperación de información mediante la metodología de análisis documental.

\section{Caracterización de la información}

La revisión documental sobre la investigación doctoral "La escritura académica en contexto: posibilidad de desarrollo profesional de los profesores universitarios" se realizó sobre la base de 50 entradas bibliográficas a las cuales se les aplicaron los criterios de pertinencia, novedad y coherencia con el tema investigado. En los trabajos revisados se destaca la actualidad en el manejo del tema, el número apropiado de fuentes bibliográficas, la rigurosidad investigativa y el espacio geográfico en el que el problema de investigación ha sido abordado (América, Canadá y Europa). Los aspectos que se tuvieron en cuenta en la caracterización del material analizado fueron: fecha de publicación, bases de datos consultadas, redes de investigación encontradas, grupos de investigación, revistas identificadas, población investigada, temáticas abordadas y metodologías de investigación utilizadas. 
Con respecto a la fecha de publicación del material indagado, se puede observar que el $100 \%$ del material se ubica en la década del 2006 al 2016, lo que permite afirmar que es un material reciente y brinda información sobre las tendencias más actuales del tema. El tipo de publicación utilizado en su mayoría son los artículos de investigación (76\%), donde se presentan los resultados que dan cuenta de los últimos avances sobre la escritura académica.

Las bases de datos consultadas fueron: Redalyc, Scielo, Aacademia, Scribd, Google Academic, Researchgate, Dialnet y Unesco, fuentes que propenden a la investigación realizada en las universidades o por investigadores reconocidos en el tema.

Las redes de investigación encontradas en la revisión fueron la Red de Lectura y Escritura de Educación Superior (REDLEES), la Red Latinoamericana de Centros y Programas de Escritura y el grupo de investigación internacional Iniciativas de Lectura y Escritura en la Educación Superior en Latinoamérica (ILEES). Con respecto a los grupos de investigación que abordan la temática de la escritura, se identificó a CONICET, de la Universidad de Buenos Aires; de Barcelona a GREAL, de la Universidad Autónoma; SINTE-LEST, de la Universidad Ramón Llull y PLURAL. De Colombia a HETEROLALIA, de la Universidad Central de Colombia; GLOTTA, de la Universidad de Ibagué; GRAPHOS, de la Universidad Sergio Arboleda.

La población investigada en un $78 \%$ corresponde a estudiantes y en un $22 \%$ a los profesores; esta información motivó en gran parte la investigación en profesores, dado que son pocos los trabajos encontrados al respecto. Las temáticas abordadas en las diferentes fuentes son: la escritura académica en la universidad, el desarrollo profesional docente, la escritura de la organización social, los géneros textuales, el panorama histórico y contrastivo de los estudios sobre lectura y escritura, y modos de leer y escribir de los universitarios.

Las metodologías de investigación utilizadas por los investigadores leídos son: descriptiva, investigación-acción, estudio de caso, revisión documental, explicativa y contrastiva, todas enmarcadas dentro de la investigación cualitativa.

\section{Desarrollo}

La revisión documental de la investigación "La escritura académica en contexto: posibilidad para el desarrollo profesional de los profesores universitarios” desarrolla tres categorías fundamentales: escritura académica, profesores universitarios y desarrollo profesional. 


\subsection{La escritura académica en la educación superior: investigaciones, prácticas y comprensiones}

La escritura "es una práctica académica y social de la cual los sujetos se apropian de manera diversa atendiendo a las circunstancias de producción, comunicación y circulación en el cual se producen" (Ortiz, 2015, p. 5); es decir, que la escritura desde una perspectiva académica debe tener en cuenta el contexto, las condiciones sociales del discurso y al emisor y receptor en sus condiciones particulares. Por tanto, la escritura académica se ve como una práctica social

llevada a cabo por miembros de una comunidad discursiva específica, y su forma misma, su contenido, y sus funciones están ligados a la naturaleza de los propósitos, las relaciones de poder, y las identidades de los participantes de esa comunidad. (Hernández, 2009, p. 21)

Por su parte, Cassany (2000) define la escritura como una manifestación de la actividad lingüística humana que implica los rasgos de intencionalidad y de contextualidad de la actividad verbal. Asimismo, constituye un hecho social que se hace manifiesto en un tiempo y en un espacio determinados y que es compartido por una comunidad específica.

La escritura académica, como práctica social, implica una serie de procesos que "conectan los pensamientos, las percepciones, las experiencias y los proyectos de la gente con colectividades más amplias de acción y creencias organizadas" (Bazerman, 2008, p.355). Los profesores usuarios de la escritura académica se encuentran enfrentados a exponer sus conocimientos, sus puntos de vista socioculturales en cada una de sus elaboraciones discursivas, y por tal razón deben asumir la escritura como un medio de comunicación entre las personas, que trasciende el tiempo y el espacio. Desde esta perspectiva, la escritura debe incorporar el sujeto escritural como un ser social, político, cultural que trasciende su ser en las palabras.

Al respecto, Hernández (2015), plantea que: “Así, en todo discurso es posible identificar posiciones frente a los eventos, frente a la organización social, a las instituciones y frente a los sujetos como miembros de grupos sociales" (p.84).

Tomando en cuenta las conexiones que se dan en la escritura, ser escritor académico supone entender la lógica de la herencia cultural, de las disciplinas científicas que involucran diferentes relaciones textuales: "la puesta en acción de conocimientos sobre las relaciones entre los textos; entre ellos y sus autores; entre los autores mismos; entre los autores, los textos y su contexto" (Lerner, 2003, p. 25). 
Desde las anteriores reflexiones investigativas, resulta muy importante proyectar en las universidades una comunidad de profesores que activen las dinámicas institucionales a través de la divulgación escrita: "Profesores que producen sus propios textos para dar a conocer sus ideas, para informar sobre hechos que los destinatarios necesitan o deben conocer, para incitar a sus lectores a emprender acciones que consideran valiosas" (Lerner, 2003, p. 26).

\subsection{Resultados}

En el análisis documental se identificaron investigaciones que centran su interés sobre cómo se conceptualiza la escritura de los profesores, los estudiantes o la comunidad académica en general, y qué estrategias utilizan para la enseñanza o el aprendizaje de la escritura.

\subsubsection{Escritura académica: conceptos y estrategias de enseñanza}

La categoría de escritura académica se revisó desde dos perspectivas: los conceptos y estrategias de enseñanza, y se examinaron los estudios de Molano y López (2006), Carlino (2006, 2007, 2009), Fernández e Izuzquiza (2010), Rincón y Gil (2010), entre otros.

Molano y López (2006), en el estudio de caso "Concepciones de profesores y estudiantes sobre la escritura académica en la universidad ICESI”, en el que los autores exploran las concepciones que tiene la ICESI sobre la escritura académica, concluyen que

las concepciones de los profesores sobre la escritura influyen directamente en las prácticas de los estudiantes. Se encontró, además, que cuando los profesores consignan comentarios pertinentes en los trabajos de sus estudiantes, éstos logran una mejor conceptualización sobre los modos de escribir. (p.119)

Carlino (2006), en el artículo "Concepciones y formas de enseñar escritura académica”, examina desde una metodología contrastiva un corpus documental de 125 universidades argentinas, australianas, canadienses y estadounidenses, para comparar las formas de ocuparse de la escritura y las concepciones que las sustentan. Las preguntas que orientan el análisis son: ¿qué se hace y dice sobre la escritura académica requerida en la universidad para los estudios de grado?, ¿se enseña a escribir como se espera?, ¿quién se ocupa de ello?, ¿está institucionalizada esta enseñanza o depende de la conciencia y voluntad individual de los docentes?, ¿cuáles son las acciones emprendidas o los sistemas instruccionales creados y con qué ideas se fundamentan? 
Carlino (2007), en la ponencia "¿Qué nos dicen las investigaciones internacionales sobre la escritura en la universidad?”, presenta una perspectiva de investigaciones sobre escritura en la universidad, cuyo centro de interés es cómo se incluyen en la enseñanza, con relación al aprendizaje de las diversas asignaturas y los resultados obtenidos en la investigación, las perspectivas de los alumnos y docentes universitarios acerca de escribir en la universidad. La autora concluye que las investigaciones sobre la escritura en la universidad han experimentado en treinta años dos tendencias concurrentes: la contextualización y la indagación sobre las relaciones entre la enseñanza que brindan las instituciones y el aprendizaje de los estudiantes.

La misma autora (Carlino, 2009b), en el libro Escribir, leer y aprender en la Universidad. Una introducción a la alfabetización académica, presenta un aporte para los maestros interesados en "disminuir la brecha entre las expectativas de los profesores y los resultados de los aprendizajes de los estudiantes durante los primeros años en el nivel superior" (p.3). El libro se centra en el desarrollo de dos interrogantes: ¿cómo se relaciona la escritura y la lectura con el aprendizaje? y ¿de qué modo pueden los docentes sacar provecho de estas relaciones en beneficio de la formación de los estudiantes? Para concluir, la autora asume la idea de responsabilidad compartida entre alumnos, docentes e institución.

Prácticas y representaciones de la escritura en la universidad: los casos de Australia, Canadá, EE-UU y Argentina es fruto de un estudio cualitativo llevado a cabo por Carlino (2009c), que contrasta la forma de la enseñanza de la escritura en universidades anglosajonas y argentinas. Como resultado, la autora identifica que las diferencias en la enseñanza de la escritura conciernen a qué se hace (cómo enseña a escribir la universidad) y cómo se le fundamenta (naturaleza y función de la escritura).

Fernández e Izuzquiza (2010), en el artículo "La escritura de textos en la universidad: una responsabilidad compartida", parten de la problemática del estudio y del aprendizaje de los estudiantes universitarios, vinculada a los procesos de deserción, rezago y fracaso académico. Desde la perspectiva del estudio, se analiza con preocupación por qué la universidad desarrolla escasas investigaciones en torno a las prácticas de enseñanza para un aprendizaje reflexivo.

El propósito de Rincón y Gil (2010), en la investigación "Las prácticas de lectura y de escritura académicas en la Universidad del Valle: tendencias", es aproximarse a los modos de leer y escribir en la Universidad del Valle. El análisis de los datos fue de naturaleza cuantitativa, lo que permitió identificar tendencias dominantes donde a los estudiantes se les solicita leer y escribir para responder a los planes de estudio. Los 
referentes teóricos de los autores se centran en los estudios de Leme Britto (2003), Becher (1993, 2001) y Carlino (2004, 2005).

Montealegre (2011), en el artículo "Leer y escribir para investigar en la educación superior: un proceso", afirma que la universidad se caracteriza por elaborar documentos académicos, sin embargo, el estudiante carece de competencias de lectura y escritura para llegar a la investigación, lo que afecta la calidad académica. El autor argumenta que algunas razones de esta situación pueden ser la falta de desarrollo investigativo y la falta de motivación de los maestros por la investigación.

Roldán, Vásquez y Rivarosa (2011), en la investigación "Mirar la escritura en la educación superior como un prisma", analizaron y compararon "las consignas de la escritura - desde las posibles tipologías y demandas cognitivas que implican en el campo particular de las ciencias biológicas" (p.2). El corpus fue tomado de tres fuentes de información: la voz de los docentes, la voz de los estudiantes y las tareas de escritura en el aula. Las autoras concluyeron que la triangulación de datos, teórica y metodológica, les permitió profundizar y dar una mirada compleja a la escritura y sus actores.

Uribe y Camargo (2011) analizan en su artículo algunas prácticas de lectura y escritura académicas en la universidad colombiana. Los autores presentan "la reflexión y el diálogo académico entre profesionales de la educación, a partir de experiencias en campos problemáticos específicos" (p.316), y concluyen que encontraron "muchas experiencias, propuestas y prácticas académicas que dan cuenta de la relevancia que ha adquirido esta problemática en los planes de estudio de las diversas carreras y posgrados" (p. 334); además, señalan que "hay pocos trabajos que ubiquen el centro de investigación en los profesores" (p.338).

Jiménez y Rojas (2012), con el artículo "La educación superior sobre la escritura y la lectura", pretenden

reflexionar sobre las implicaciones que tiene la consideración de lectura y la escritura como prácticas socioculturales, el papel que estas prácticas desempeñan en las diversas actividades que se desarrollan en el contexto universitario y las tendencias investigativas sobre su abordaje. (p. 19)

Los autores concluyen que aunque la lectura y la escritura se conciban como prácticas propias de los contextos universitarios, "ellas deben ser objeto de problematización y acción reflexiva, en particular, en relación con los propósitos y responsabilidades sociales que la universidad cumple a través de la gestión del conocimiento" (2012, pp. 32-33). 
Camps y Castelló (2013), en el estudio "La escritura académica en la universidad", analizan diferentes aportes que se han hecho sobre la escritura académica, a partir de los géneros que se producen en la universidad con el propósito de otorgar sentido y significado a las prácticas de escritura de los estudiantes, y presentan reflexiones sobre el uso y la enseñanza de los géneros en la docencia universitaria.

Carlino (2013) presenta en el artículo "Alfabetización académica diez años después", "las experiencias y publicaciones latinoamericanas sobre la enseñanza de y con la lectura y la escritura en la universidad en la última década” (p.355). Además, analiza las iniciativas desarrolladas en Argentina y muestra la evolución del concepto de alfabetización académica.

Pérez y Rincón (2013), en el libro ¿Para qué se lee y se escribe en la universidad colombiana?, presentan el proceso de investigación cuyo propósito principal es "describir, caracterizar, analizar e interpretar las prácticas de lectura y escritura académicas en la universidad colombiana, con el fin de proponer unas orientaciones de política al respecto" (p.41). A manera de conclusión indican que "Aunque no fue posible identificar unas directrices explícitas de políticas institucionales al respecto las universidades colombianas sí están preocupadas por el tema de la lectura y la escritura académicas" (p.277).

Carlino, Iglesia y Lavalt (2013), en la investigación "La escritura académica en la universidad", examinan las prácticas declaradas y las concepciones de lectura y escritura que pueden inferirse de ellas, a partir de un estudio entre profesores terciarios argentinos. Las autoras centran el interés en describir las acciones que se realizan para ayudar a afrontar las dificultades de los alumnos para leer comprensivamente y escribir con claridad. Como conclusión, las autoras manifiestan que "lo más provechoso para el aprendizaje es entramar o tejer el trabajo sostenido de lectura y escritura con la enseñanza de cada materia al servicio del aprendizaje de sus propios contenidos" (p.128).

Soler (2013), en el libro Usted ya en la universidad y no saber escribir, toma como hilo conductor, de la investigación presentada, la escritura, el poder y la exclusión. La metodología parte del análisis del discurso para "dar cuenta de las situaciones de inclusión y exclusión presentes en los procesos de escritura académica en el contexto universitario seleccionado" (p.13). Además, reitera que "se requiere una educación basada en la diversidad, que luche contra todas las formas de exclusión, no solo étnicas, sino sociales, culturales y educativas" (p.132).

Cárdenas (2014), con su artículo "Prácticas de lectura y escritura en educación superior: lo que los estudiantes y docentes dicen de la lectura y la escritura en las 
licenciaturas de una universidad en el caribe colombiano", resultado de una investigación realizada en diez licenciaturas, tuvo el propósito de "describir, interpretar y comprender las prácticas de lectura y escritura que se llevan a cabo en esas licenciaturas" (p.6o). Este estudio tuvo como base teórica la alfabetización académica. La metodología fue cualitativa - etnográfica.

Salazar (2015) identifica: "las diferentes líneas de investigación que se han planteado para el estudio de la escritura en el contexto de la educación superior" (p.1), en su artículo "La escritura académica universitaria: diferentes perspectivas de estudio". La metodología utilizada fue la revisión literaria desde tres dimensiones de estudio de la escritura: lingüística, cognitiva y social. El autor presenta como conclusión las diferentes líneas de investigación que tienen como propósito "explicar y comprender que en la escritura académica universitaria convergen en tres grandes dimensiones de investigación: dimensión lingüística, cognitiva y social” (p.16).

Bazerman et al. (2016), en el artículo "Panorama histórico y contrastivo de los estudios sobre lectura y escritura en educación superior en América Latina”, plantean que "los estudios sobre lectura y escritura en educación superior cuentan con al menos dos décadas de desarrollo en Latinoamérica. Sin embargo, aún son escasas las investigaciones sistemáticas sobre la configuración de este espacio disciplinar” (p.100). Las conclusiones obtenidas evidencian que "una gran mayoría de artículos presentan interés en la investigación empírica de la escritura. Se abordan entornos y géneros diversos, aunque con predominancia en formación de grado y el área de las Humanidades y las Ciencias Sociales" (p. 120).

Chois y Jaramillo (2016), en "La investigación sobre la escritura en posgrado: estado del arte", realizan el análisis de 52 documentos. Los autores afirman que en el marco de la educación posgradual la escritura tiene un lugar fundamental por lo menos en dos sentidos: "por un lado, porque es una importante herramienta de aprendizaje, y por otro, porque la apropiación de las formas de escribir de una comunidad académica determinada permite el ingreso y la participación activa de los novatos en ella” (p.228).

Arroyo y Gutierrez (2016) realizan un estudio cualitativo sobre competencias escritoras en la formación universitaria del profesorado y sobre sus hallazgos manifiestan que "la práctica regular de la escritura en la universidad no es suficiente para mejorar la escritura de los estudiantes y, por lo tanto, es necesario organizar la enseñanza académica en la escritura en los niveles universitarios" (p. 145); es decir, que la formación académica en el pregrado no garantiza que un profesor sea escritor, para 
lograrlo es necesario profundizar en los elementos propios de la escritura académica o incluir dentro de los programas de formación pregradual.

Guerra (2016), en la investigación "Peer support in small group EFL writing tasks" plantea que las investigaciones han demostrado que, al escribir con un par, los estudiantes se retroalimentan en elementos formales y discursivos de la lengua. Es decir, que la lectura y coevaluación de un texto por un compañero puede posibilitar una mejor redacción de textos.

Rojas (2016), en la investigación "Género en lingüística sistémico funcional y en sociorretórica: apuntes para una didáctica de la lectura y la escritura en la universidad”, manifiesta que la escritura como proceso académico "no es posible delegar en los docentes de las disciplinas la responsabilidad en la formación de lectores y escritores, pues ellos no poseen un conocimiento especializado sobre la dinám ica del lenguaje" (p.96); puesto que la escrita es un proceso de reflexión y estructuración mental complejo que exige un conocimiento especializado.

Piñeros, Orjuela y Torres (2018) afirman:

La relevancia innegable que tiene la escritura en la vida social, académica y secular de los ciudadanos, su adquisición, dominio y puesta en práctica, se relaciona directamente con la producción científica y académica de un país y, por ende, con su avance educativo, político, democrático y social (p.176).

En otras palabras, la escritura constituye la socialización del sujeto, quien, a su vez, construye la sociedad.

Así, los textos escritos no pueden separarse de los contextos sociales de interacción, son parte de un lenguaje contextualmente situado. Esta perspectiva tiene sus bases en los estudios socioculturales, donde se ha caracterizado la escritura como una práctica situada. Por tanto, la escritura académica se ve como una práctica social que es realizada por "personas que pertenecen a una comunidad discursiva específica y su forma su contenido, y sus funciones están ligados a la naturaleza de los propósitos, las relaciones de poder y las identidades de los participantes de esa comunidad" (Zamora, 2009, p. 21).

Por útimo, es necesario reconocer que hace parte de la misión de la universidad compartir el conocimiento científico, los nuevos saberes, las prácticas sociales y su quehacer; así se conforma la comunidad del conocimiento. Entonces, la escritura académica no es solamente una práctica social individual, "sino que los usos de la escritura surgen de lo que se hace con ella en determinadas comunidades discursivas, es 
decir, en conjuntos de gente que comparten actividades, conocimientos, valores y que emplean la escritura para determinados fines" (Carlino, 2005, p.9).

\subsubsection{La escritura académica media la actuación y el desempeño del profesor universitario}

En la presente investigación, otra categoría muy importante es el profesor universitario, por lo tanto se expondrán las disposiciones que lo caracterizan, los retos que debe asumir y el contexto actual en el cual debe desarrollarse profesionalmente, a partir de los siguientes teóricos: Marcelo (2002), Imbernón (2000), Zabalza (2009), Patiño (2006) y Mas (2011), entre otros.

Los profesores universitarios, como lo plantea Zabalza (2009), son personas que ponen en práctica una profesión y que es necesario asumirlas desde dos roles: persona y profesor: "No somos profesores, o trabajadores a secas. Somos personas que desarrollan su actividad profesional en la docencia universitaria. Olvidarlo produce desajustes y tensiones que en nada favorecen el buen ejercicio profesional" (p.72). En otras palabras, la esencia de la persona del profesor enmarca su desarrollo profesional, así que no se puede desconocer ninguna de estas dimensiones.

Patiño (2006) señala que "el profesor universitario de hoy es producto de un sistema que no ha utilizado la escritura como estrategia de aprendizaje y, por tanto no ha podido incorporarla y reconocerla como una didáctica en el ejercicio docente" (p.127). Por tanto, se asume desde esta propuesta investigativa que la escritura académica media la actuación y desempeño del profesor universitario.

Formar al profesor universitario en búsqueda de su desarrollo profesional es uno de los intereses centrales de las universidades, y al respecto Nóvoa (2009) plantea que "es imposible definir un buen profesor" (p.206); sin embargo, determina una serie de disposiciones con la cuales debe contar un buen profesor: el conocimiento, cultura profesional, tacto pedagógico, dimensiones colectivas y de colaboración, del trabajo en equipo, de la intervención conjunta en los proyectos educativos y compromiso social. Para alcanzar estas disposiciones es necesario que el profesor se forme continuamente, nutra su persona y su profesión a través de un desarrollo profesional reflexivo, consensuado y contextualizado.

El profesor en ejercicio cada vez es más consciente de la necesidad de su formación, por tal razón se está acentuando la idea de que "la responsabilidad de la formación recae cada vez más en los propios profesionales. Hacer de nuestras escuelas 
espacios en los que no sólo se enseña sino en los que los profesores aprenden, representa el gran giro que necesitamos" (Marcelo, 2002, p.29); esta es una necesidad presente en el contexto actual universitario, donde cada día un complejo contexto social está promoviendo "la importancia del sujeto, la participación en las decisiones y las vertiginosas transformaciones tecnológicas y de los medios de comunicación" (Imbernón, 2000, p. 37). Estas presiones sociales exigen un nuevo modelo de profesor universitario "que no se conforme con dominar la materia científica, sino que sepa unir a la investigación y a la gestión, el cada vez más relevante papel de la docencia y de emociones y en aspectos sociales y ambientales” (Imbernón, 2000, p. 37).

En el caso colombiano, la preocupación es muy alta dado que, según Patiño (2006), el personal docente universitario está formado, en la mayoría de los casos "por profesionales de diferentes áreas que establecen un compromiso educativo con las instituciones universitarias sin recibir preparación docente previa. Los profesionales dedicados a la docencia se ven obligados a enfrentar su quehacer desde una experiencia empírica" (p.127). Y resulta, en ocasiones, muy difícil superar el arraigado y viejo supuesto que afirma que un buen profesor universitario es aquel que domina la materia científica, ya que ese conocimiento le da la habilidad por sí mismo para enseñarla ( Imbernón, 2000).

Mas (2011) afirma que ante los nuevos retos de la universidad, algunas de la funciones que debe desempeñar el profesor universitario son: orientar aprendizajes, investigar, crear equipos multidisciplinares y participar de la gestión de la organización, entonces, saber solo el campo disciplinar no es suficiente para un profesional que se dedica a la educación.

1.2.3 La escritura académica en contexto, posibilidad para el desarrollo profesional de los profesores de educación superior

El desarrollo profesional de los profesores (Ávalos, 2007; Domínguez, 2007; Marín, 2014 e Imbernón 2006) como proceso continuo de aprendizaje, tiene como propósito fortalecer al profesor en su profesión, a partir de la reflexión y participación activa de este en el análisis de su progreso, el cual debe ser desarrollado durante toda su vida. De ahí la urgencia y el desafío de generar acciones formativas en contexto, tal como lo propone Nárvaez (2011), "Definir las prioridades de la formación del maestro universitario en contextos colombianos, e incluso latinoamericanos, en relación con la escritura en la universidad, es un debate por iniciar" (p. 121).

La formación docente se puede entender "como un proceso que ocurre a través de (sic) toda la vida de un profesor o profesora" (Ávalos, 2007, p. 78); desde este punto de vista, la persona que decida ser profesor debe comprender que todos los días debe estar 
aprendiendo y reflexionando sobre su quehacer. Por su parte, Domínguez (2007) afirma que la formación debe contribuir "a fortalecer en el educador su fe en sí mismo, su capacidad mental y espiritual, su sentido de reflexión y participación, su motivación al logro de las metas propuestas, su visión para la resolución de problemas y su espíritu de servicio" (p.6o); en efecto, la formación docente debe incidir en la experiencia vital y profesional del profesor para lograr la trascendencia de lo aprendido en el proceso de formación.

Por otra parte, Imbernón (2006), en el marco de una conferencia, afirmó: “yo le digo siempre al profesorado sobre la formación inicial, tienen que introducir en los maestros la idea de que es el principio, y que van a tener que continuar formándose toda su vida" (p. 5); es decir, que la experiencia de ser profesor es para toda la vida, así como su formación.

La formacion docente se materializa en todas las posibilidades de aprender a las que se ve enfrentado el profesor, y estas deben ser organizadas e intencionadas a partir de la reflexión de los profesores para avanzar en el desarrollo profesional. En palabras de Day (2005):

El desarrollo profesional consiste en la totalidad de las experiencias naturales de aprendizaje y de aquellas actividades conscientes y planificadas dirigidas intencionalmente al beneficio de individuos, grupos o escuelas, que contribuyen a la calidad de la educación en el aula. (p.15)

El rol del profesor en el proceso de formación debe ser el de un "sujeto de formación" (Imbernón, 2006, p.7), esto es, que reflexiona sobre su aprendizaje, prevé el impacto que dicha formación tendrá en su profesión y tiene un papel activo en su formación.

Atendiendo a la necesidad de formación profesional de los profesores, la Unesco (2009), en la Conferencia Mundial sobre la Educación Superior - la nueva dinámica de la educación superior y la investigación para el cambio social y el desarrollo-, manifestó que la educación superior debe ampliar la formación de docentes: "tanto inicial como en el empleo, con planes y programas de estudio que den a los docentes la capacidad de dotar a sus alumnos de los conocimientos y las competencias que necesitan en el siglo XXI" (p.3); en otros términos, hay que repensar la formación profesional desde dos factores: "un elemento personal y el externo" (Ávalos, 2007, p.78). El primero consiste en el interés de aprender y el compromiso total con la enseñanza y su profesión, es decir, en cuanto su propósito vital de ser profesor, esto es, su voluntad personal; el segundo es un elemento externo, que se ocasiona por las actividades de formación organizadas y su 
relación con las necesidades de las instituciones educativas a las que pertenecen los profesores, podría entenderse como la oportunidad de aprender (Ávalos, 2007).

Desde los dos factores, internos y externos, el desarrollo profesional de los profesores, según Marín (2014), “debe ser visible, reconocido, promovido, prestigiado y debe ser asumido por todos/as, en la cotidianidad (de las instituciones), por ser este parte integral de los valores de sus miembros e intente el desarrollo organizativo de la institución" (p.126); de esta manera, la profesionalización docente alcanzará la trascendencia para gestar el cambio en las instituciones y en la educación de hoy. Entonces, corresponde a las instituciones formadoras de profesores identificar los mecanismos necesarios para que el profesor pueda identificar sus propias debilidades y contribuir, en forma eficiente, en el marco de esta investigación, al desarrollo de las habilidades escriturales (Domínguez, 2007)

Vaillant (2007), desde la revisión literaria "Mejorando la formación y el desarrollo profesional docente en latinoamerica", señala que "las nuevas expectativas y los desafíos a los que se enfrentan las sociedades y economías sitúan a la educación en el centro del debate y a los docentes como actores clave" (p.1). En el estudio, la autora concluye que los docentes importan para "influir en el aprendizaje de los alumnos y para mejorar la calidad de la educación. Importan en definitiva como un recurso imprescindible para las sociedad del conocimiento" (p.15).

Carlino (2009a), en el capítulo "Desarrollo profesional de docentes para leer y escribir a través del currículum”, examina la experiencia de formación en servicio, muestra las opciones pedagógicas asumidas por el equipo formador, y analiza las funciones de la lectura y la escritura" (p.51). Los marcos de referencia de la autora son los estudios de Bazerman (2001), Britton (1994), Gee (1996), Lerner (2003). Este trabajo de análisis y puesta en marcha es ejecutado desde la metodología de investigaciónacción.

Cartolari y Carlino (2009), en las Memorias del I Congreso Internacional de Investigación y Práctica Profesional en Psicología, presentaron la revisión bibliográfica "Formación docente, concepciones y prácticas de lectura y escritura", donde reseñaron 50 publicaciones. En la bibliografía analizada, las autoras hallaron tres fundamentos para incluir la enseñanza de la lectura y la escritura en la educación terciaria: "como procesos cognitivos necesarios para el aprendizaje, como prácticas socialmente situadas de apropiación de modos de hacer y pensar en las disciplinas y como instrumentos políticos para acrecentar las oportunidades de acceso y participación social de los alumnos" (p.206). 
Narváez (2010), en el artículo "Escritura académica y formación de docentes universitarios: reflexiones a partir del recorrido por algunas experiencias”, presenta el análisis de cuatro experiencias de formación de docentes universitarios. Entre los hallazgos, la autora identificó la concurrencia en los tópicos de discusión: "la escritura pública y privada; el vínculo entre la escritura, la investigación y la trayectoria intelectual de los autores; y, la escritura como tarea prescrita para los profesores” (p.113).

Cartolari y Carlino (2012), en el artículo "Leer y escribir en la formación docente: aporte en las investigaciones anglosajonas", realizan una revisión de 49 investigaciones anglosajonas sobre leer y escribir en la formación de docentes, publicadas entre $1985 \mathrm{y}$ 2010. En palabras de las autoras, el análisis de los atículos les permitió analizar aportes coincidentes y divergentes con respecto a los siguientes aspectos: "concepciones y prácticas [...], cómo enseñar a leer y escribir en las disciplinas, usos de la lectura y la escritura en la enseñanza e iniciativas institucionales para transformar creencias y prácticas alfabetizadoras de los profesores [...]” (p.6).

Laco, Natale y Ávila (2012), en el libro La lectura y la escritura en la formación académica, docente y profesional, presentan un ejercicio de formación en lectura y escritura a través del programa de tutorías que inició en el año 2006, con el cual se fortalece a los profesores y estudiantes para que asuman la lectura y la escritura como un enfoque y metodología habitual de trabajo. Las autoras realizaron un encuentro de la lectura y la escritura en la formación académica, docente y profesional, con el propósito de contribuir a la capacitación de los profesores de las diferenctes disciplinas; fruto de este encuentro es este libro con las memorias de las ponencias presentadas. La conclusión central es la necesidad de crear una red latinoamericana de investigadores o instituciones que trabajen para apoyar los procesos de lectura y escritura en la formación.

Núñez y Poch (2014) en el artículo "Lectura y escritura: trazando historias en la formación profesional docente", presentan la discusión sobre la importancia de una "neoalfabetización que permita abrir los canales de la lecto escritura como estrategias de profesionalización para los profesores" (p.303). Los autores concluyen que es muy importante que los profesores desarrollen un nivel de consciencia de la práctica, del conocimiento y del trabajo colaborativo para alcanzar las metas del aprendizaje. Se privilegia la transposición, desde la interacción, los desafíos conjuntos y de coconstrucciones de sentido.

\section{Conclusiones}

En esta revisión documental se pudo observar que las investigaciones analizadas centran su atención en: 
- Las dificultades que pueden existir en el proceso escritor.

- El profesor universitario no tiene formación escritural.

- Escribir es una tarea compleja, los profesores universitarios no están formados apropiadamente para ser usuarios de la escritura y hay pocas investigaciones con respecto al uso de la escritura académica.

- Las explicaciones a los problemas de lectura y escritura en el estudiante, dejando de lado a los profesores.

- En el aprendizaje de los estudiantes y la didáctica de los profesores.

Los anteriores trabajos analizados contextualizan la situación de los profesores universitarios, profesionales de diversas áreas que se ven comprometidos a cumplir con otras funciones además de la docencia, tales como la investigación, la proyección social y algunas actividades administrativas que les exigen, fuera de "dar clase", desempeñarse en actividades donde la escritura se convierte en una "práctica social" (Vargas, 2013, p.76), transversal para su desarrollo profesoral.

$\mathrm{Al}$ respecto, la investigación de donde se deriva el presente artículo centra su interés en la escritura académica en contexto: posibilidad para el desarrollo profesional de los profesores universitarios; es decir, que el profesor será el sujeto implicado y la escritura se asume como una posibilidad sociocultural que promoverá el desarrollo profesional del profesor.

\section{Referencias}

Arroyo, R. A. \& Gutiérrez-Braojos, C. G. (2016). Competencias escritoras en la formación universitaria del profesorado. Revista Electrónica Interuniversitaria de Formación del Profesorado, 19(3), 135-147. Recuperado de http://doi.org/10.6018/reifop.19.3.221191

Ávalos, B. (2007). El desarrollo profesional continúo de los docentes: lo que nos dice la experiencia internacional y de la región latinoamericana. Revista Pensamiento Educativo, 41(2), 77-99. Recuperado de http://pensamientoeducativo.uc.cl/files/journals/2/articles/417/public/417-9341-PB.pdf

Bazerman, C. (2008). La escritura de la organización social y la situación alfabetizada de la cognición: extendiendo las implicaciones sociales de la escritura de Jack Goody. Revista Signos, 41, 355-380. Recuperado de http://www.scielo.cl/pdf/signos/v41n68/art01.pdf 
Bazerman, C. (2012). Actos de habla, géneros y sistemas de actividades: de qué manera los textos organizan las actividades y grupos sociales. Benemérita Universidad Autónoma de Puebla, 122-136. Recuperado de https://lecturayescrituraunrn.files.wordpress.com/2013/o8/bazerman-unidad1.pdf

Bazerman, C., Navarro, F., Ávila, N., Tapia, M., Vera, L. Moritz, M. \& Narváez, E. (2016). Panorama histórico y contrastivo de los estudios sobre lectura y escritura en educción superior en américa Latina. Revista Signos, Estudios de Lingüística, 49(1) 100-126. http://dx.doi.org/10.4067/So718-09342016000400006

Camps, A. \& Castelló, M. (2013). La escritura académica en la universidad. Revista de Docencia Universitaria, 11(1), 17-36. Recuperado de http://redu.net/redu/files/journals/1/articles/579/public/579-2168-1-PB.pdf

Cárdenas, L. (2014). Prácticas de lectura y escritura en educación superior: lo que los estudiantes y docentes dicen de la lectura y la escritura en las licenciaturas de una universidad en el caribe colombiano. Revista Praxis, 10, 60-77. Recuperado de www.revistas.unimagdalena.edu.co/index.php/praxis/article/download/1359/768

Carlino, P. (2006a). Concepciones y formas de enseñar escritura académica. Un estudio contrastivo. Signo \& Seña, 16, 71-117. Recuperado de

https://media.utp.edu.co/referenciasbibliograficas/uploads/referencias/articulo/carlin o2006concepcionesyformasdeensenarescrituraacademicapdf-4mY5k-articulo.pdf

Carlino, P. (2006b). La escritura en la investigación. (Documento de trabajo, 19). Recuperado de https://www.aacademica.org/paula.carlino/66.pdf

Carlino, P. (2007). ¿Qué nos dicen las investigaciones internacionales sobre escritura en la universidad? Revista Cuadernos de Psicopedagogía, 4, 21-40. Recuperado de https://www.aacademica.org/paula.carlino/131.pdf

Carlino, P. (2009a). Desarrollo profesional de docentes para leer y escribir a través del currículum. En Lectura y escritura, un asunto de todos. Revista acta Académica, 140. Recuperado de: https://www.aacademica.org/paula.carlino/140

Carlino, P. (2009b). Escribir, leer y aprender en la Universidad. Una introducción a la alfabetización académica. España: Fondo de Cultura Económica.

Carlino, P. (2009c). Prácticas y representaciones de la escritura en la universidad: los casos de Australia, Canadá, EE-UU y Argentina. Revista Cuadernos de Pedagogía Universitaria, $\quad 12, \quad 6-17 . \quad$ Recuperado de https://www.aacademica.org/paula.carlino/189.pdf 
Carlino, P. (2013). Alfabetización académica diez años después. Revista Mexicana de Investigación Educativa, 18(57), 355-381. Recuperado de http://www.redalyc.org/pdf/140/14025774003.pdf

Carlino, P., Iglesia, P. \& Lavalt, I. (2013). Concepciones y prácticas declaradas de profesores terciarios en torno al leer y escribir en las asignaturas. Revista de Docencia Universitaria, 11, 105-135. Recuperado de http://redu.net/redu/files/journals/1/articles/583/public/583-2172-1-PB.pdf

Cartolari, M. \& Carlino P. (2009). Formación docente, concepciones y prácticas de lectura y escritura una revisión bibliográfica. En Trabajo presentado en el I Congreso Internacional de Investigación y Práctica Profesional en Psicología. XVI Jornadas de Investigación. Quinto encuentro de investigadores en Psicología del Mercosur. Universidad de Buenos Aires, Facultad de Psicología. Recuperado de https://www.aacademica.org/paula.carlino/10o.pdf

Cartolari, M. \& Carlino P. (2012). Leer y escribir en la formación docente: aportes de las investigaciones anglosajonas. Revista Acción Pedagógica, 21, 6-17. Recuperado de http://www.saber.ula.ve/bitstream/123456789/36641/1/dossiero1.pdf

Chois, P. \& Jaramillo, L. (2016). La investigación sobre la escritura en posgrado: estado del arte. Revista Lenguaje, 44, 227-259. Recuperado de http://www.scielo.org.co/pdf/leng/v44n2/v44n2a05.pdf

Cassany, D. (2000). Construir la escritura. Barcelona: Paidós. Recuperado de https://es.scribd.com/doc/142990604/Cassany-Construir-la-escritura-pdf\#

Day, C. (2005). Formar docentes: cómo, cuándo y en qué condiciones aprende el profesorado. Madrid: Narcea. Recuperado de

https://books.google.com.co/books?id=WN2NQA-

$\mathrm{k} 7 \mathrm{GwC} \&$ printsec $=$ frontcover\&dq=christopher + day + formar+docentes+desarrollo + profesional\&hl=es\&sa $=$ X\&ved $=$ oahUKEwiJh42ZkM3SAhWBwiYKHbTdCAsQ6 AEIGjAA\#v=onepage\&q=christopher\%20day\%2oformar\%2odocentes\%20desarr ollo\%2oprofesional\&f=fal

Domínguez de Rivero, M. (2007). El maestro y la escritura desde la perspectiva de la formación docente. Sapiens, Revista Universitaria de Investigación, 8(2), 57-65. Recuperado de http://www.redalyc.org/pdf/410/41080204.pdf

Fernández, G. \& Izuzquiza, M. (2010). La escritura de textos en la universidad: una responsabilidad compartida. Buenos Aires: Universidad Nacional del Centro de la Provincia de Buenos Aires. Recuperado de http://www.feeye.uncu.edu.ar/web/ posjornadasinve/area3/Lengua\%20-\%20Didactica\%20de\%20la\%2olengua\%20- 
\%20TICs/263\%20-\%2oFernandez\%20y\%2oIzuzquiza\%20\%20UN\%20Ctro \%20Prov\%20Bs\%20As.pdf

Galeano, M. (2012). Estrategias de investigación social cualitativa: el giro en la mirada. Medellín: La Carreta.

Guerra, J. (2016). Peer Support in Small Group EFL Writing Tasks. Revista Cuadernos de Lingüística Hispánica, (28), 149-166. Recuperado de http://revistas.uptc.edu.co/ index.php/linguistica_hispanica/article/view/4913/4317

Hernández, G. (2009). Escritura académica y formación de maestros. ¿Por qué no acaban las tesis? México: Tiempo de Educar. Recuperado de www.redalyc.org/pdf/311/31113164002.pdf

Hernández, N. (2015). La construcción mediática de perdedores y vencedores en el campo político: análisis de las metáforas, tópicos y lexicalización. Revista Cuadernos de Lingüística Hispánica, (25), 81-98. Recuperado de http://www.scielo.org.co/pdf/clin/n25/n25ao5.pdf

Imbernón, F. (2000). Un nuevo profesorado para una nueva universidad ¿conciencia o presión? Revista Interuniversitaria de Formación del Profesorado, 38, 37-46. Recuperado de

https://www.researchgate.net/publication/39138715_Un_nuevo_profesorado_para_un a_nueva_universidad_conciencia_o_presion

Imbernón, F. (2006). Actualidad y nuevos retos de la formación permanente. Revista Electrónica de Investigación, 8(2), 1-11. Recuperado de http://www.scielo.cl/pdf/estped/v40nEspecial/art18.pdf

Jiménez, H. \& Rojas, G. (2012). La educación superior desde la escritura y la lectura. Revista Amazonia Investiga, 1, 19-35. Recuperado de www.udla.edu.co/revistas/index.php/amazonia-investiga/article/view/3/5

Lerner, D. (2003). Leer y escribir en la escuela: lo real, lo posible y lo necesario. México: Fondo de Cultura Económica. Recuperado de https://coleccion.siaeducacion.org/sites/default/files/files/9-lernerdelia_2003leer_y_escr.pdf

Marcelo, C. (2002). Los profesores como trabajadores del conocimiento. Certidumbre y desafíos para una formación a lo largo de la vida. Revista Educar, 30, 27-56. Recuperado de www.raco.cat/index.php/Educar/article/download/207 
Laco, L. Natale, L. \& Ávila, M. (2012). La lectura y la escritura en la formación académica, docente y profesional. Argentina: Universidad Nacional de General Sarmiento. Recuperado de www.ungs.edu.ar/.../Laco-L.-Natale-L.-y-Ávila-M.2012.-La-lectura-y-la-escritura-en-la-formacion-academica-docente-yprofesional.pdf

Manjarrés, M. \& Vargas, G. (1998). La investigación educativa sobre la infancia en el Distrito Capital. Informe sobre el estado del arte (1986-1996). Colombia: Pontificia Universidad Javeriana.

Marín, M. (2014). El desarrollo de los profesores: un asunto por abordar en el centro educativo. Revista Sophia, 1O(1), 107-122. Recuperado de http://revistas.ugca.edu.co/files/journals/1/articles/223/public/223-647-1PB.html

Mas, O. (2011). El profesor universitario: sus competencias y formación. Revista de Currículum y Formación del Profesorado, 15(3), 195-211. Recuperado de http://www.ugr.es/ recfpro/rev153COL1.pdf

Molano, L. \& López, G. (2006). Concepciones de profesores y estudiantes sobre la escritura académica en la Universidad Icesi. Revista Lenguaje, 35, 119-146. Recuperado https://media.utp.edu.co/referenciasbibliograficas/uploads/referencias/articulo/ 823concepciones-de-profesores-y-estudiantes-sobre-la-escritura-academica-enla-universidad-icesipdf-vrIt1-articulo.pdf

Montealegre, A. (2011). Leer y escribir para investigar en la educación superior: un proceso. Revista Actualidades Pedagógicas, 199-212. Recuperado de https://revistas.lasalle.edu.co/index.php/ap/article/view/548

Narváez, E. (2010). Escritura académica y formación de docentes universitarios: reflexiones a partir del recorrido por algunas experiencias. Revista Pedagogía y Saberes, 33, 113-124. Recuperado de

http://webcache.googleusercontent.com/search?q=cache:PjjYPtf8vYJ:revistas.pedagogi ca.edu.co/index.php/PYS/article/download/759/733+\&cd=1\&hl=es\&ct=clnk\&gl $=\mathrm{co}$

Nóvoa, A. (2009). Para una formación de profesores construida desde la profesión. Revista de Educación, (350), 203-218. Recuperado de http://www.revistaeducacion.mec.es/re350/re350_09.pdf

Núñez, M. \& Poch, P. (2014). Lectura y escritura: trazando historias en la formación profesional docente. Revista Estudios Pedagógicos, 4O(1), 303-321. Recuperado de: http://www.scielo.cl/pdf/estped/v40nEspecial/art18.pdf 
Ortiz, E. (2015). La escritura académica en el contexto universitario. Revista del Instituto de Estudios en Educación Universidad del Norte, 22, 1-16. Recuperado de: http://www.scielo.org.co/pdf/zop/n22/n22ao2.pdf

Patiño, L. (2006). La escritura académica en la formación del docente universitario. Revista Educación y Pedagogía, 18(46), 127-133. Recuperado de https://dialnet.unirioja.es/servlet/articulo?codigo $=2362546$

Pérez, M. \& Rincón, G. (2013). ¿Para qué se lee y se escribe en la universidad colombiana? Un aporte a la consolidación de la cultura académica del país. Colombia: Colciencias. Recuperado de https://wac.colostate.edu/books/colombian/highered.pdf

Piñeros, A., Orjuela, D. \& Torres, A. (2018). Una mirada crítica a las prácticas escriturales del grado quinto de la básica primaria. Revista Cuadernos de Lingüística Hispánica, (31), 173-190. Recuperado de http://revistas.uptc.edu.co/index.php/linguistica_hispanica/article/view/7763/6 145

Rincón, G. \& Gil, J. (2010). Las prácticas de lectura y de escrituras académicas en la Universidad del Valle: tendencias. Revista Lenguaje, 38(2), 387-419. Recuperado de http://daca.univalle.edu.co/politica_curricular/Practicas-de-lectura-y-deescrituraacademicas-en-la-univalle-tendencias.pdf

Rojas, I. (2016). Género en lingüística sistémico funcional y en sociorretórica. Apuntes para una didáctica de la lectura y la escritura en la universidad. Revista Cuadernos de Lingüística Hispánica, (28), 93-114. Recuperado de http://revistas.uptc.edu.co/index.php/linguistica_hispanica/article/view/4910

Roldán, C. Vásquez, A. \& Rivarosa A. (2011). Mirar la escritura en la educación superior como un prisma. Revista Iberoamericana de Educación, 55(3), 1-13. Recuperado de

http://rieoei.org/rie_contenedor.php?numero=4010\&titulo=Mirar\%2ola\%20esc ritura\%20en\%20la\%20educaci\%C3\%B3n\%20superior\%20como\%20un\%20prism a

Salazar, C. (2015). La escritura académica universitaria: diferentes perspectivas de estudio. Revista Diálogos sobre Educación, 11, 1-21. Recuperado de http://www.scielo.org.ar/scielo.php?script=sci_arttext\&pid=S1995$77852016000100010 \& \operatorname{lng}=$ es\&nrm=iso

Soler, S. (2013). Usted ya en la universidad y no saber escribir. Escritura y poder en la universidad. Bogotá: Universidad Distrital Francisco José de Caldas. Recuperado 
de

www.die.udistrital.edu.co/sites/...ud/.../usted_ya_en_la_universidad_y_no_sab er_escribir.pdf

Unesco. (2009). Conferencia Mundial sobre la Educación Superior. La nueva dinámica de la educación superior y la investigación para el cambio social y el desarrollo. París: Unesco. Recuperado de http://www.unesco.org/education/WCHE2009/comunicado_es.pdf

Uribe, G. \& Camargo, Z. (2011). Prácticas de lectura y escritura académicas en la universidad colombiana. Revista Internacional de Investigación en Educación Magis, 3, 317-341. $\quad$ Recuperado de http://www.redalyc.org/pdf/2810/281021734005.pdf

Vaillant, D. (2007). Mejorando la formación y el desarrollo profesional docente en Latinoamérica. Revista de Pensamiento Educativo, 41(2), 1-16. Recuperado de http://www.ub.edu/obipd/PDF\%2odocs/Formaci\%C3\%B3\%2oPermanent/Educ aci\%C3\%B3\%2oPrimaria/Publicacions/Mejorando\%2ola\%2oformaci\%C3\%B3n\% 20y\%20el\%2odesarrollo\%2oprofesional\%2odocente\%2oen\%2oLatinoam\%C3\%A 9rica.\%20Vaillant,D.pdf

Vargas, A. (2013). Revisión entre iguales, esritura académica e identidad en la formación docente en una universidad colombiana. (Tesis doctoral). Universitat Pompeu Fabra. Recuperado de http://www.tdx.cat/bitstream/handle/10803/125115/tavf.pdf?sequence=1

Zabalza, M. (2009). Ser profesor universitario hoy. Revista La Cuestión Universitaria, 5, 69-81. Recuperado de http://tecnologiaedu.us.es/mec2011/htm/mas/3/31/47.pdf

Zamora, G. (2009). Escritura académica y formación de maestros ¿por qué no acaban las tesis? Revista Tiempo de Educar, 10(19,) 11-40. Recuperado de http://www.redalyc.org/pdf/311/31113164002.pdf 